\title{
Elecciones 2009 en Uruguay: permanencia de lealtades políticas y accountability electoral
}

\begin{tabular}{c}
\hline \hline Lucía Selios \\
Instituto de Ciencia Política \\
Faculdade de Ciencias Sociales \\
Universidad de la República \\
Daniela Vairo \\
Instituto de Estudos Sociais e Políticos \\
Universidade Estadual do Rio de Janeiro \\
\hline \hline
\end{tabular}

\begin{abstract}
Resumen: En este artículo se indagan las principales características asociadas al comportamiento electoral en las elecciones nacionales uruguayas en el año 2009. Através de la estimación de modelos probit en base a una encuesta electoral, se encuentra que la evaluación de la gestión del gobierno, la percepción de la economía y los aspectos ideológicos aparecen jugando fuertemente en esta elección caracterizada por la baja volatilidad. Estos hallazgos indican la existencia de fuertes lealtades a los partidos agrupados en familias ideológicas, combinadas con aspectos evaluativos sobre el desempeño económico y político, que pautan el voto de los uruguayos en esta elección.
\end{abstract}

Palabras-clave: comportamiento electoral; opinión pública; elecciones; Uruguay

\begin{abstract}
This paper inquires about the main characteristics of voting behavior in uruguayan national elections in 2009. Using a probit analysis based on an election poll it is found that government management, perception of economy and ideological issues play an important role in this election characterized by low volatility. These findings indicate strong party loyalties aggregated in "ideological families" as well as evaluative aspects about political and economic performance that determine the Uruguayan vote.
\end{abstract}

Keywords: voting behavior; public opinion; elections; Uruguay 


\section{Introducción ${ }^{1}$}

Las elecciones de octubre de 2009 Ilevaron por segunda vez consecutiva al Frente Amplio a la Presidencia de la República. En dichos comicios se constató el menor nivel de volatilidad desde 1984 pero, al mismo tiempo, investigaciones recientes (DE ARMAS, 2009; SELIOS, 2009) insinúan un cambio en los factores que explican el voto que llevarían a una reinterpretación del comportamiento electoral en el país. En este artículo indagaremos las principales características asociadas al voto en el año 2009 y a partir de ellas intentaremos descifrar los principales cambios electorales y su proyección.

El análisis del comportamiento electoral de los votantes uruguayos en las últimas elecciones nacionales será realizado desde la óptica de la evolución de las fidelidades partidarias (ROSE \& Mc ALLISTER, 1990). Los autores presentan un modelo que permite observar el fenómeno electoral en la relación simbiótica partido-elector, considerando las principales teorías sobre comportamiento electoral e interpretando los resultados en función de la estabilidad o inestabilidad en los votantes y los partidos políticos.

Concretamente, este artículo se centra en la parte del modelo de análisis que se enfoca desde el lado del elector, identificando una variedad de lealtades que son construidas a "lo largo de la vida" y que influyen en el comportamiento del ciudadano común el día de la elección. De esta manera, el modelo incluye: “...la influencia de los padres durante el crecimiento de sus hijos; el interés material socio. económico que los adultos desarrollan en el trabajo y como dueños de casa; los valores políticos que ofrecen medios duraderos para evaluar lo que una persona experimenta o conoce; el contexto social de la comunidad; la religión y la nación; y el desempeño actual de los partidos y sus líderes"2 (ROSE \& Mc ALLISTER, 1990, p.1).

Así, el peso de las diferentes lealtades en la decisión electoral permite determinar si el electorado en general presenta un comportamiento estable o en su defecto, inestable: cuando la decisión está sujeta a evaluaciones de la coyuntura política, económica y la oferta de los partidos en cada elección.

\section{El marco analítico: relación partidos-electores como marco interpretativo}

Variada literatura sobre comportamiento electoral argumenta que los votantes no deciden su voto sólo por las propuestas de los partidos sino que existe una decisión razonada íntimamente, ligada a los procesos vividos por los individuos y sus expectativas sobre los partidos políticos. Se entiende que en algunas situaciones el elector no es pasivo ni estrictamente racional, pero sí reflexivo y con capacidad para replantearse el voto, particularmente cuando existe "disonancia cognitiva" (OSKAMP, 1991),

\footnotetext{
${ }^{1}$ Este artículo modifica y mejora el análisis ya presentado por las autoras en un capítulo que forma parte del libro BUQUET y JOHNSON (2010) bajo el nombre: "Desde las lealtades partidarias a la accountability electoral: Análisis de las determinantes del voto y la reciente evolución electoral en Uruguay". Agradecemos la invalorable colaboración para este artículo de la Licenciada en Ciencia Política Guadalupe Goyeneche y el Comunicador Esteban Selios. Asimismo, agradecemos especialmente los comentarios del árbitro anónimo de la revista.

2 Traducción de las autoras.
} 
OPINIÃO PÚBLICA, Campinas, vol. 18, n 1, Junho, 2012, p. $197-215$

producida por cambios en las orientaciones de los partidos políticos que no son acompañadas por las propias convicciones y características del elector.

Una línea argumentativa similar, señala que el votante no es un agente racional que se comporta como un "consumidor" ante un producto en competencia como postula la escuela economicista del voto (DOWNS, 1957), sino que a partir de las ofertas, el votante proyecta su voto como una inversión para su bienestar futuro (POPKIN, 1994). En esa decisión evalúa elementos prospectivos y retrospectivos $^{3}$ de los partidos, las políticas y los discursos (FIORINA, 1981; POWELL, 2000; MARAVALL y SANCHEZ CUENCA, 2008).

Fiorina (1981) desarrolla el concepto de voto retrospectivo, donde la decisión de voto se explica por la evaluación que realiza el votante sobre la performance del gobierno anterior, utilizando una lógica de premios y castigos, en la cual se premia a los gobiernos con los que se está satisfecho y se castiga a los partidos que no han realizado una buena gestión gubernamental. De esta manera, el comportamiento electoral puede ser interpretado a través de los juicios retrospectivos que realiza el electorado acerca de los resultados de las acciones del gobierno. Con esta lógica Fiorina (1981) concluye que lo que determina la elección del votante son las expectativas que tiene sobre cada uno de los candidatos, aunque estas expectativas se encuentran claramente afectadas por las experiencias pasadas.

Otras vertientes desarrollan una explicación del comportamiento electoral fundada en teorías acerca de las lealtades partidarias y pertenencia partidaria. (LAZARSFELD, BERELSON y GAUDET, 1948), en su trabajo pionero en esta perspectiva, afirman que las personas que comparten ciertas características estructurales generalmente votan por el mismo candidato, generando homogeneidad política en los grupos sociales. Esas características vienen dadas por variables estructurales tales como la edad, el status socioeconómico, el lugar de residencia, la ocupación o la religión.

En este trabajo se le dará especial atención al modelo que presentan (ROSE \& Mc ALLISTER, 1990). Los autores consideran que el fenómeno electoral debe tener en cuenta dos tipos de actores operando: los partidos por un lado y los electores por otro. Los autores proponen cuatro posibles relaciones lógicas resultantes de la interacción entre partidos y electores en función del cambio o estabilidad de cada uno.

En el caso de los electores, la inestabilidad indica que su decisión electoral se basa en su evaluación de los resultados de las políticas, de la gestión de gobierno y de la oposición, etc. Por su parte, la estabilidad se visualiza cuando los electores continúan votando por su partido más allá de los resultados, coyuntura política y estrategias partidarias (ROSE \& Mc ALLISTER, 1990, p.29). En este caso su voto se asocia a características socioeconómicas, demográficas, el llamado "voto clasista", el "voto religioso", etc. Cuando los electores son inestables, los elementos prospectivo y retrospectivo del voto juegan un papel muy importante porque en estas evaluaciones de promesas y acciones partidarias se funda la base de la representación democrática, permitiendo la accountability (rendición de cuentas) que implica que el elector tiene la capacidad de castigar o premiar a los políticos por su actuación pasada; es

\footnotetext{
3 Las teorías elaboran este punto sobre expectativas programáticas vs. resultados de políticas, predominando el plano
} económico. 
SELIOS, L.; VAIRO, D. Elecciones 2009 en Uruguay: permanencia de lealtades...

la capacidad de remover políticos según su performance. Esta noción implica que de algún modo el político es responsable ante el electorado, quien evalúa sus promesas pasadas y sus resultados.

La estabilidad del comportamiento electoral de los ciudadanos, se sustenta en las variables que se aprenden tempranamente en la vida de los individuos y, por lo tanto, son más difíciles de cambiar. Específicamente las dimensiones que indicarían la estabilidad de los electores en el modelo de (ROSE \& Mc ALLISTER, 1990) provienen originalmente del trabajo de (LAZARSFELD, BERELSON y GAUDET, 1948).

El hecho de que los electores sean estables no significa que no se produzcan cambios en el sistema político, pero estos son lentos y progresivos. Por ejemplo, en la teoría de clivajes, propuesta por Lipset y Rokkan en 1967, se considera que el comportamiento electoral refleja características sociales o de clase (KRIESI, 1998; PARAMIO, 1998) que en el tiempo produjo cambios en varios sistemas políticos. Así, si los votantes de clase trabajadora votan a un partido y éste siempre recibe los votos de ese grupo social, conforme aumenten los trabajadores aumentará, elección tras elección, su base electoral independientemente de la coyuntura política o los liderazgos. Para que estas explicaciones socio. estructurales de las lealtades políticas y el voto tengan sentido, deben existir partidos políticos capaces de generar y capitalizar estas diferencias en la sociedad.

De esta manera, el partido es el que "propone y los electores disponen" (ROSE \& Mc ALLISTER, 1990, p.2) porque son los primeros quienes tienen la capacidad e incentivos para cambiar la orientación y estrategias hacia un electorado por el que compiten. Si los electores son estables, las acciones de los partidos estarán principalmente ${ }^{4}$ orientadas a reforzar las identidades políticas, las características socio estructurales y activando otros clivajes. Ahora bien, cuando los electores son inestables la competencia electoral pasa al dominio de otras estrategias electorales: el partido en el gobierno realiza un discurso sobre los logros alcanzados y promete mejorar el bienestar a través de sus políticas, mientras que la oposición critica las acciones del gobierno y realiza promesas electorales sobre las ideas para su gestión. Así, en los casos en que el modelo de ROSE \& Mc ALLISTER (1990) prevé electores inestables opera mejor la accountability electoral.

En definitiva, siguiendo los enfoques teóricos de los autores reseñados y especialmente de ROSE \& MC ALLISTER, la propuesta del artículo es analizar el comportamiento electoral en los comicios presidenciales de 2009 en Uruguay.

\section{El caso: los cambios del sistema de partidos y el comportamiento electoral en Uruguay La evolución electoral}

A lo largo de su historia democrática Uruguay presenta un sistema de partidos enraizados con electorados estables (GONZÁLEZ, 1993; 1999; SOTELO, 1999). De hecho, el primer gran cambio en el Uruguay fue la alternancia de 1958, cuando el Partido Nacional alcanza el gobierno nacional gracias a la

\footnotetext{
${ }^{4}$ Los partidos no son maquinarias homogéneas y en todos los casos conviven estrategias que combinan elementos afectivos y simbólicos con promesas electorales y críticas a la gestión. El tema aquí es la prioridad en la estrategia hacia su electorado.
} 
OPINIÃO PÚBLICA, Campinas, vol. 18, n 1, Junho, 2012, p. $197-215$

unificación partidaria y a un contexto de descontento generalizado. El segundo gran cambio es en 1971 con la irrupción del Frente Amplio ${ }^{5}$ y el consiguiente cambio en el sistema de partidos.

En la primera elección después a la dictadura militar, en 1984, el mapa electoral resurge casi incambiado. El Partido Colorado gana la Presidencia, seguido por el Partido Nacional y por último el Frente Amplio con similares guarismos a los de 1971. En la siguiente elección de 1989 aparecen dos novedades. Por un lado, vuelve la alternancia en el gobierno; el Partido Nacional gana las elecciones y traza importantes acuerdos legislativos y de gobierno con parte del Partido Colorado. Por su parte, el Frente Amplio accede al gobierno municipal de Montevideo. En 1994 triunfa el Partido Colorado aunque sus contingentes legislativos son más escasos, lo que lleva al primer gobierno de coalición en el país (CHASQUETTI, 1998).

La siguiente elección en 1999 se produce bajo la normativa de la reforma constitucional de 1996, que introdujo novedades que hacen a la lógica de competencia inter e intra-partidaria: mayoría absoluta o segunda vuelta y candidatura única por partido para la elección presidencial, separación de elecciones nacionales y departamentales, internas partidarias previas obligatorias y simultáneas, eliminación de acumulación por sublemas en la elección de Diputados, entre otras disposiciones. En esta elección la izquierda gana la primera vuelta pero al no conseguir la mitad mas uno de los votos debió disputar la segunda vuelta con el Partido Colorado, partido que resultó vencedor gracias a los votantes del Partido Nacional (CANZANI, 2000, p.256).

En la elección de 2004 la izquierda accede al gobierno y con mayoría legislativa propia, confirmando una tendencia en el comportamiento electoral detectada varios años antes (GONZÁLEZ \& QUEIROLO, 2000). En esta elección también se verifica una drástica reducción de la fragmentación del sistema de partidos que se ubica en un formato de dos partidos y medio. En base a este hecho muchos autores (GONZÁLEZ \& QUEIROLO, 2000; LANZARO, 2000; MOREIRA, 2004; LUNA, 2004; BUQUET \& DE ARMAS, 2004; CHASQUETTI \& GARCÉ, 2005); señalan que el bipartidismo tradicional ha encontrado su nueva versión entre dos bloques o familias ideológicas: "tradicional y desafiante". En 2004 el trasiego de votantes entre bloques es mucho menor al registrado entre partidos (BUQUET, 2005), y ambos se reducen drásticamente en 2009. En la última elección, parece que se ha hallado una suerte de equilibro entre los votantes de los distintos bloques y el traspaso dentro de la familia tradicional se mantiene alto (Tabla 1).

Tabla 1

Resultados electorales, volatilidad en Uruguay 1984-2009

\begin{tabular}{|l|l|l|l|l|l|l|l|}
\hline & & 1984 & 1989 & 1994 & $1999 *$ & $\mathbf{2 0 0 4}$ & $\mathbf{2 0 0 9}$ \\
\hline \multirow{2}{*}{ Volatilidad } & Interpartidaria & 5,7 & 13,4 & 11,5 & 9,9 & 24,8 & 7,6 \\
\cline { 2 - 8 } & Interbloque & 4,9 & 7,1 & 5,6 & 8,5 & 9,4 & 1,7 \\
\hline
\end{tabular}

Fuente: Banco de Datos Política y RRII Facultad de Ciencias Sociales, Universidad de la República.

\footnotetext{
${ }^{5}$ Este partido, que se denominó por mucho tiempo "coalición de izquierda", fue la unión de todos los grupos de izquierda y
} algunos escindidos de los partidos tradicionales. 
SELIOS, L.; VAIRO, D. Elecciones 2009 en Uruguay: permanencia de lealtades...

En síntesis, esta evolución electoral, que determinó un movimiento de un sistema bipartidista a uno multipartidista, derivó en la conformación de dos polos o bloques políticos. Por una parte, la familia tradicional compuesta por el Partido Colorado y el Partido Nacional, partidos fundacionales ubicados del centro a la derecha del espectro político ideológico. Por su parte, la familia de izquierda compuesta actualmente por el Frente Amplio, partido ubicado del centro hacia la izquierda de la escala ideológica.

\section{Enfoques desde los partidos y desde los electores}

Quienes han estudiado el comportamiento electoral de los uruguayos a lo largo del tiempo han aportado elementos de gran utilidad para la comprensión del fenómeno desde el punto de vista de los partidos y de los electores. En estos trabajos se evidencia la existencia de las familias o bloques políticos, no sólo en las preferencias electorales sino también en las actitudes de los votantes y las estrategias de los partidos.

En cuanto al estudio de los partidos, se han desarrollado algunas interpretaciones que procuran explicar el cambio en la competencia política en el país. En primer lugar, el cambio ideológico del Frente Amplio, que en los últimos años avanza hacia el centro del espacio político (GARCÉ \& YAFFÉ, 2004), al mismo tiempo que opera un movimiento hacia la derecha por parte de los partidos tradicionales (ALTMAN, 2002). En segundo lugar, la "crisis de intermediación", particularmente en los partidos tradicionales (MIERES, 1997), tema que sido también problematizado por Yaffé (2005) y Luna (2004), que coinciden al afirmar que en los últimos años los partidos tradicionales pierden su capacidad de establecer clientelas o bases electorales amplias y estables.

Y finalmente la interpretación que hace a los liderazgos, competencia interna y campañas. Aquí se destaca la importancia de los liderazgos y la competencia interna como estrategia exitosa para los partidos así como el manejo de la campaña, buscando equilibrios de competencia y cooperación para el fortalecimiento partidario (YAFFÉ, 2005; CHASQUETTI \& GARCÉ, 2005).

Desde el punto de vista de los electores, que es en el que nos centraremos en la parte de análisis de datos de este artículo, se han desarrollado numerosos trabajos que procuran explicar el comportamiento de los votantes. Las explicaciones van desde los componentes socio-estructurales hasta los valores políticos y la ideología, la evaluación del desempeño económico y de liderazgos y los efectos de las campañas en el voto. A continuación haremos un breve repaso de estas visiones sobre el comportamiento electoral.

En los enfoques de tipo socio-estructural se recorren varias estrategias analíticas. Una de ellas es analizar los resultados electorales y las características del territorio y la población en términos agregados (MIERES, 1994; MOREIRA, 2004; MAGRI, 2004) Estos trabajos identificaron una "modernización" y "tradicionalización" del voto en el país; allí dónde las características de la población eran de tipo "modernas" el voto al Frente Amplio era mayoritario, mientras que en las "tradicionales" ganaba el Partido Nacional o el Colorado.

Otros autores trabajan con encuestas e identifican el peso de las tradiciones familiares, que aparecían como relevantes entre los frentistas y no así entre votantes de los partidos tradicionales, quienes habían perdido la capacidad de trasmisión familiar del voto. Es decir, los hijos de 
OPINIÃO PÚBLICA, Campinas, vol. 18, no 1, Junho, 2012, p. $197-215$

frenteamplistas votaban al Frente Amplio en una proporción mucho mayor que los de las familias tradicionales (MONESTIER, 1999; MOREIRA, 2000). Por su parte la tesis demográfica explicaba el crecimiento electoral del Frente Amplio a través del recambio generacional. Ésta expone que mayoritariamente, los nuevos electores votaban por el Frente Amplio, mientras que las generaciones mayores preferían a los partidos tradicionales. (AGUIAR, 2000; CANZANI, 2000).

Dentro de los enfoques que explican el comportamiento electoral a partir de las posiciones y grupos sociales, se destaca aquel que se centra en el llamado "voto religioso". A principios de la década de 1990 el voto vinculado a la religión aparecía como relevante para diferenciar a los electorados de los partidos Nacional y Colorado. Finalmente, otros trabajos analizan aspectos socioeconómicos destacando que los frentistas pertenecerían a clases de ingresos medios y altos con educación media y alta y más jóvenes (MIERES, 1990; 1994).

Investigaciones recientes encuentran que la conexión entre voto y regiones más o menos "tradicionales" en Uruguay parecería haber desaparecido (BUQUET, 2005). También se erosiona la relación entre clase, voto, y "modernidad" en el voto a la izquierda (MOREIRA, 2005). Y, finalmente, tiene fin el efecto demográfico en el voto al Frente Amplio (DE ARMAS, 2009) y con ello la trasmisión familiar del voto.

Por otra parte se ha elaborado un enfoque basado en los valores políticos que ha sido ampliamente desarrollado y caracteriza a los frenteamplistas como demócratas insatisfechos (ROSELL, 2002; MOREIRA, 2004; HARETCHE, 2004).

Dentro de este enfoque, los análisis de autoidentificación ideológica son muy utilizados para la explicación del comportamiento electoral y existe una fuerte correspondencia entre la posición de los electores y la del partido elegido (COLOMER \& ESCATEL, 2005). Este hecho es especialmente destacado en Uruguay y considerando movimientos en la distribución del electorado identificados ante la contienda electoral (GONZÁLEZ, 2004), se pudieron realizar estimaciones muy precisas de los resultados de las elecciones de 2004 y 2009 en base a encuestas de opinión pública (BUQUET \& SELIOS, 2004; SELIOS, 2009).

Luego de las elecciones de 2004 surgen algunos trabajos que incorporan el peso de los liderazgos y el desempeño del gobierno (CANZANI, 2005; CHASQUETTI \& GARCÉ, 2005) y otros en la línea de la performance económica (LUNA, 2002; ARMELLINI, 2005) como determinantes del voto. En estos últimos se analiza cómo ciertos indicadores económicos objetivos, tales como el desempleo, explican cambios en las opiniones de los votantes. En esta línea se constata además que en los meses de implementación del Impuesto a la Renta de las Personas Físicas las consultoras de opinión pública marcan una caída en la intención de voto al Frente Amplio que se mantuvo hasta mediados del 2009 (SELIOS, 2009).

Por último, la mayoría de los trabajos sobre comportamiento electoral en Uruguay estiman que los efectos de los medios y de la campaña tienen un peso relativamente bajo en la decisión de voto. Sin embargo, en un trabajo reciente De Armas (2005) investiga el momento de decisión del voto durante la campaña en 1994 y 2004 y encuentra que el porcentaje de encuestados que declaran haberse decidido 
SELIOS, L.; VAIRO, D. Elecciones 2009 en Uruguay: permanencia de lealtades...

durante la campaña coincide con la volatilidad registrada en cada elección. Además descubre que los votos de los "activados, conversos, o movilizados" se distribuyeron de manera asimétrica, siendo la campaña del Frente Amplio la que menos impactos tuvo en el electorado. El momento de decisión de voto durante la campaña parece haber influido en el traspaso de votos colorados a nacionalistas, o sea que el impacto se produce dentro del bloque o familia tradicional, pero es marginal entre bloques (DE ARMAS, 2005).

En definitiva, el recorrido histórico y las interpretaciones del voto en el país indican que recientemente los partidos, tomados como bloques o familias, se movieron en sus posicionamientos ideológicos, pero al mismo tiempo perdieron ciertas capacidades de intermediación y construcción de identidades políticas. Al mismo tiempo, las clásicas interpretaciones del comportamiento electoral en Uruguay han perdido vigencia pues pierden fuerza explicativa las variables estructurales y cobran fuerza las explicaciones sobre la evaluación de la gestión y la economía.

En esta coyuntura, y colocándonos del lado de los electores, cabe preguntarse entonces: ¿cuáles son las determinantes del voto a las familias ideológicas en Uruguay para las elecciones de 2009?

\section{Metodología}

Para el análisis de las determinantes del voto a las dos familias ideológicas, se utiliza la base de datos $^{6}$ de la segunda ola de una encuesta panel telefónica realizada por el Instituto de Ciencia Política en octubre y noviembre de 2009. El análisis se realiza a través de la estimación de dos modelos estadísticos probit $^{7}$, uno correspondiente a los determinantes del voto en 2009 a la familia de izquierda ${ }^{8}$ y otro a la familia tradicional ${ }^{9}$.

Los posibles determinantes del voto pueden dividirse en varias dimensiones explicativas del comportamiento electoral. De esta manera, las variables independientes incluidas en ambos modelos contemplan las principales teorías sobre el voto y la acumulación de la literatura nacional.

Las 6 dimensiones abarcan entonces varios ángulos de interpretación del voto en el país: componentes sociodemográficos, relaciones particularistas, comportamientos o hábitos políticos, importancia de valores políticos, satisfacción de intereses y efectos de campaña. El resultado de la operacionalización de las variables utilizadas se presenta en la Tabla 2:

\footnotetext{
${ }^{6}$ Encuesta panel (dos mediciones) realizada en el marco del taller Procesos Electorales de la Licenciatura en Ciencia Política de la Facultad de Ciencias Sociales, UdelaR. Se trata de una encuesta telefónica representativa de todo el país con muestreo probabilístico aleatorio polietápico por conglomerados con tres puntos de muestreo con afijación proporcional a la distribución de la población: nivel nacional, departamental y local. El marco de muestreo fue el listado de teléfonos georeferenciados, que tiene amplia cobertura en el Uruguay (95\%). El margen de error es de $+.4,1 \%$, con $95 \%$ de confianza. La base de datos que se utiliza en este trabajo pertenece a la segunda ola (604 casos realizados entre el 7 y el 15 de noviembre de 2009, en relación a los 916 casos de la primera ola de octubre). Si bien la mortandad muestral entre la primera y la segunda medición es de $34 \%$, se constató que la segunda muestra, con la que trabajamos aquí, está ajustada a criterios poblacionales y fue ponderada por intención de voto.

${ }^{7}$ Los modelos probit son parte de los modelos de respuesta dicotómica. En este caso, los modelos probit explican la probabilidad de votar a la familia de izquierda (modelo 1) y de votar a la familia tradicional (modelo 2) en función de ciertas variables independientes seleccionadas.

${ }^{8}$ La variable de voto a la familia de izquierda asume el valor 1 cuando el encuestado declara haber votado al Frente Amplio en octubre de 2009 y valor 0 para cualquier otra respuesta.

${ }^{9}$ La variable de voto a la familia tradicional asume el valor 1 cuando el encuestado declara haber votado al Partido Nacional o al Partido Colorado y valor 0 para cualquier otra respuesta.
} 
OPINIÃO PÚBLICA, Campinas, vol. 18, no 1, Junho, 2012, p. $197-215$

Tabla 2

\begin{tabular}{|c|c|c|c|}
\hline Dimensión & Sub-dimensión & Variable & Nomenclatura y Tratamiento \\
\hline \multirow{5}{*}{$\begin{array}{l}\text { Componentes } \\
\text { socio-económicos }\end{array}$} & \multirow{3}{*}{ Básicas } & Sexo & $\begin{array}{l}\text { Mujer asume el valor } 1 \text { si el encuestado es mujer y } \\
0 \text { si es hombre. }\end{array}$ \\
\hline & & Edad & $\begin{array}{l}\text { Recodificada en tres tramos, y fueron introducidos } \\
\text { en el modelo los tramos de } 30 \text { a } 64 \text { años (variable } \\
\text { de30a64) y } 65 \text { años y más (variable de65ymas). }\end{array}$ \\
\hline & & Religión & $\begin{array}{l}\text { Nocreyen asume el valor } 1 \text { si la persona responde } \\
\text { que no es creyente en Dios y valor } 0 \text { para el resto } \\
\text { de las respuestas. }\end{array}$ \\
\hline & Posición social & Nivel educativo & $\begin{array}{l}\text { Recodificada en tres categorías, y fueron } \\
\text { introducidas en el modelo: secundar, que asume } \\
\text { el valor } 1 \text { si el encuestado alcanzó el nivel } \\
\text { educativo secundario y primaria, que asume el } \\
\text { valor } 1 \text { si alcanzó el nivel primario. }\end{array}$ \\
\hline & Contextual & $\begin{array}{l}\text { Lugar de } \\
\text { residencia }\end{array}$ & $\begin{array}{l}\text { Montevideo asume el valor } 1 \mathrm{si} \text { el encuestado } \\
\text { reside en Montevideo y valor } 0 \text { si reside en el } \\
\text { interior. }\end{array}$ \\
\hline \multirow{3}{*}{$\begin{array}{l}\text { Relaciones } \\
\text { particularistas }\end{array}$} & Influencias de su entorno & $\begin{array}{l}\text { Homogeneidad } \\
\text { voto del entorno } \\
\text { familiar y amigos }\end{array}$ & $\begin{array}{l}\text { Homogene asume el valor } 1 \text { si el encuestado } \\
\text { declara que todos o la mayoría de su familia y que } \\
\text { todos o la mayoría de sus amigos votaron en la } \\
\text { elección de } 2009 \text { el mismo partido que el } \\
\text { encuestado. }\end{array}$ \\
\hline & Fidelidad del voto & $\begin{array}{l}\text { Voto al mismo } \\
\text { partido }\end{array}$ & $\begin{array}{l}\text { Siempre/ asume el valor } 1 \text { si el encuestado } \\
\text { manifiesta haber votado siempre al mismo partido } \\
\text { en todas las elecciones en las que participó y valor } \\
0 \text { para el resto de las respuestas. }\end{array}$ \\
\hline & Involucramiento político & Activismo político & $\begin{array}{l}\text { Activismo asume el valor } 1 \text { cuando el encuestado } \\
\text { asistió a algún acto, caravana o reunión política } \\
\text { durante la última campaña. }\end{array}$ \\
\hline $\begin{array}{l}\text { Comportamientos } \\
\text { o hábitos políticos }\end{array}$ & Interés en política & Interés en política & $\begin{array}{l}\text { Intpol asume el valor } 1 \text { si el encuestado está muy } \\
\text { o bastante interesado en política y } 0 \text { si está poco } \\
\text { o nada interesado o no responde. }\end{array}$ \\
\hline \multirow[b]{2}{*}{$\begin{array}{l}\text { Importancia de } \\
\text { valores políticos }\end{array}$} & Estado/Mercado & $\begin{array}{l}\text { Intervención del } \\
\text { Estado }\end{array}$ & $\begin{array}{l}\text { Interven asume el valor } 1 \text { cuando el encuestado } \\
\text { considera que el Estado uruguayo debería } \\
\text { aumentar su intervención en la economía y } 0 \text { para } \\
\text { quienes creen que debe disminuir o no responden. }\end{array}$ \\
\hline & Ideología & $\begin{array}{l}\text { Autoidentificación } \\
\text { ideológica }\end{array}$ & $\begin{array}{l}\text { Se recodificó en tres categorías y se introdujeron } \\
\text { en el modelo: autoidiz, que asume el valor } 1 \\
\text { cuando el encuestado se coloca en los valores } \\
\text { entre } 0 \text { y } 3 \text { de la escala de autoidentificación } \\
\text { ideológica (denominada izquierda) y } \\
\text { autoidder, que asume el valor } 1 \text { cuando el } \\
\text { encuestado se ubica en los valores denominados } \\
\text { de derecha (del } 7 \text { al } 10 \text { en la escala). }\end{array}$ \\
\hline \multirow{3}{*}{$\begin{array}{l}\text { Satisfacción de } \\
\text { intereses }\end{array}$} & $\begin{array}{l}\text { Evaluación de Gestión del } \\
\text { Gobierno }\end{array}$ & $\begin{array}{l}\text { Evaluación de } \\
\text { Gestión del } \\
\text { Gobierno }\end{array}$ & $\begin{array}{l}\text { Gestiong asume el valor } 1 \text { si el encuestado califica } \\
\text { a la gestión de gobierno de Vázquez como buena } \\
\text { o muy buena y } 0 \text { para el resto de las respuestas }\end{array}$ \\
\hline & $\begin{array}{l}\text { Evaluación de la } \\
\text { situación económica país }\end{array}$ & $\begin{array}{l}\text { Evaluación de } \\
\text { situación } \\
\text { económica actual } \\
\text { y futura del país }\end{array}$ & $\begin{array}{l}\text { Econpais asume valor } 1 \text { si el encuestado } \\
\text { manifiesta que la situación económica actual del } \\
\text { país es buena o muy buena y al mismo tiempo } \\
\text { considera que la situación económica del país } \\
\text { dentro de un año será mejor. }\end{array}$ \\
\hline & $\begin{array}{l}\text { Evaluación de la } \\
\text { situación económica } \\
\text { persona }\end{array}$ & $\begin{array}{l}\text { Evaluación de } \\
\text { situación } \\
\text { económica actual } \\
\text { y futura personal }\end{array}$ & $\begin{array}{l}\text { Econpers asume valor } 1 \text { si el encuestado } \\
\text { manifiesta que su situación económica actual } \\
\text { personal es buena o muy buena y al mismo } \\
\text { tiempo considera que dicha situación dentro de un } \\
\text { año será mejor. }\end{array}$ \\
\hline \multirow{2}{*}{$\begin{array}{l}\text { Efectos de } \\
\text { campaña }\end{array}$} & \multirow{2}{*}{$\begin{array}{l}\text { Información política y } \\
\text { propaganda }\end{array}$} & $\begin{array}{l}\text { Influencia de } \\
\text { información } \\
\text { política en } \\
\text { campaña }\end{array}$ & $\begin{array}{l}\text { Impinf asume el valor } 1 \text { si el encuestado cree que } \\
\text { la información política le ayudó mucho o bastante } \\
\text { para formar su opinión sobre los candidatos y } 0 \\
\text { para el resto de las respuestas. }\end{array}$ \\
\hline & & $\begin{array}{l}\text { Influencia de } \\
\text { propaganda }\end{array}$ & $\begin{array}{l}\text { Infpub asume el valor } 1 \text { si el encuestado señala } \\
\text { que las propagandas políticas de la campaña } \\
\text { influyeron mucho o bastante en su decisión de } \\
\text { voto. }\end{array}$ \\
\hline
\end{tabular}

Fuente: Elaboración propia 


\section{Comprendiendo las determinantes del voto: estimación de modelos y análisis Voto a la familia de izquierda}

¿Qué variables están asociadas con el voto a la familia de izquierda? En la Tabla 3 (Modelo 1) se presenta la estimación de los cambios en la probabilidad de que el encuestado manifieste haber votado a la familia de izquierda en octubre de 2009. Además de los coeficientes, su signo y su significación se presentan los efectos marginales de las variables que resultaron estadísticamente significativas, como forma de dar cuenta de la magnitud del efecto de las variables independientes sobre la probabilidad de que los encuestados respondan que votaron a la familia de izquierda.

Tabla 3. Modelo $1^{10}$

Coeficientes y efectos marginales, variable dependiente fliaizq

\begin{tabular}{|l|l|l|}
\hline Variables & Coeficiente & $\begin{array}{l}\text { Efectos marginales sobre } \\
\text { P(fliaizq } 1)\end{array}$ \\
\hline Mujer & $0.274(0.174)$ & \\
\hline De30a64 & $0.204(0.224)$ & \\
\hline De65ymas & $0.468(0.277)$ & \\
\hline Nocreyen & $0.192(0.186)$ & \\
\hline Secundar & $0.246(0.182)$ & \\
\hline Primaria & $-0.065(0.246)$ & \\
\hline Montevid & $0.516^{* * *}(0.172)$ & $0.202(0.066)$ \\
\hline Siemprel & $-0.122(0.182)$ & \\
\hline Intpol & $-0.016(0.171)$ & \\
\hline Homogene & $0.775^{* * *}(0.198)$ & $0.301(0.072)$ \\
\hline Activism & $0.622^{* * *}(0.181)$ & $0.244(0.069)$ \\
\hline Intervene & $0.663^{* * *}(0.167)$ & $0.251(0.062)$ \\
\hline Autoidiz & $0.760^{* * *}(0.210)$ & $0.295(0.076)$ \\
\hline Autoidde & $-1.469^{* * *}(0.231)$ & $-0.493(0.055)$ \\
\hline Gestiong & $1.359^{* * *}(0.185)$ & $0.491(0.055)$ \\
\hline Econpais & $0.547^{* * *}(0.182)$ & $0.215(0.070)$ \\
\hline Econpers & $0.439^{* *}(0.193)$ & $0.174(0.075)$ \\
\hline Impinf & $-0.269(0.189)$ & \\
\hline Infpub & $0.310(0.268)$ & \\
\hline
\end{tabular}

Fuente: Elaboración propia

-Errores estándar entre paréntesis; ***, **, significativa al 99 y $95 \%$, respectivamente.

Las variables que tienen una relación significativa con la variable dependiente y presentan un efecto marginal más fuerte, corresponden a la dimensión de valores políticos y satisfacción de intereses.

Concretamente, en cuanto a la satisfacción de intereses, el modelo predice que evaluar positivamente la gestión del gobierno aumenta en 49 puntos porcentuales la probabilidad de votar al

10 La probabilidad predicha por el modelo de que los encuestados respondan que votaron al Frente Amplio (fliaizq=1) es de 0,453 . Al tratarse de una variable dependiente binaria, esta probabilidad significa que en promedio un $45,3 \%$ de los entrevistados respondería que votó al Frente Amplio. Mientras tanto, el porcentaje efectivo de personas que en la muestra manifestaron haber votado al Frente Amplio es de 47,9\%, por lo que la diferencia entre el valor predicho y el efectivo es pequeña $(2,6 \%)$. Por tanto, dicha medida de bondad de ajuste del modelo es satisfactoria aunque es una medida parcial. 
OPINIÃO PÚBLICA, Campinas, vol. 18, n 1, Junho, 2012, p. $197-215$

Frente Amplio. Además, quienes tienen una visión positiva sobre la situación económica actual y futura, tanto personal como del país, tienden a votar en mayor medida al FA con relación a quienes tienen visiones negativas o no emiten opinión. El modelo estima un aumento de 22 puntos porcentuales en la probabilidad de votar al FA para quienes tienen una percepción positiva sobre el país y de 17 puntos porcentuales cuando se evalúa positivamente la situación personal.

Con respecto a los valores políticos, la probabilidad de que los individuos voten al Frente Amplio en 2009 disminuye 49 puntos porcentuales si el entrevistado se autoidentifica ideológicamente como de derecha. En cambio, si el entrevistado se identifica como de izquierda, la probabilidad del voto al Frente Amplio aumenta en 30 puntos porcentuales. Asimismo, considerar que debe aumentar la intervención del Estado en la economía aumenta 25 puntos porcentuales la probabilidad de votar al Frente Amplio.

Según los resultados del modelo, también influyen algunas variables de la dimensión comportamental y de relaciones particularistas. Así, el voto a la familia de izquierda se vincula positivamente también con la participación en actos, caravanas o reuniones políticas (aumenta 24 puntos porcentuales la probabilidad estimada del voto al Frente Amplio) y con presentar homogeneidad entre el voto personal y el de los familiares y amigos (la probabilidad estimada aumenta 30 puntos porcentuales).

Finalmente, dentro de las variables de la dimensión sociodemográfica el modelo predice que vivir en Montevideo aumenta la probabilidad estimada de votar al Frente Amplio en 20 puntos porcentuales, con respecto a quienes viven en el interior.

\section{Voto a la familia tradicional}

¿Y qué variables están asociadas con el voto a la familia tradicional? En la Tabla 4 (Modelo 2) se presenta la estimación de los cambios en la probabilidad de que el encuestado manifieste haber votado a la familia tradicional en las últimas elecciones nacionales, dadas las mismas variables independientes que en el modelo para la familia de izquierda. 
SELIOS, L.; VAIRO, D. Elecciones 2009 en Uruguay: permanencia de lealtades...

Tabla 4. Modelo $2^{11}$

Coeficientes y efectos marginales, variable dependiente fliatrad

\begin{tabular}{|c|c|c|}
\hline Variables & Coeficiente & $\begin{array}{l}\text { Efectos marginales } \\
\text { sobre } P(\text { fliatrad }=1)\end{array}$ \\
\hline mujer & $.0 .108(0.171)$ & \\
\hline de30a64 & $.0 .627^{* * *}(0.224)$ & $-0.244(0.085)$ \\
\hline de65ymas & $-0.899 * * *(0.281)$ & $-0.316(0.083)$ \\
\hline nocreyen & $-0.518 * *(0.203)$ & $-0.194(0.071)$ \\
\hline secundar & $0.030(0.182)$ & \\
\hline primaria & $0.121(0.240)$ & \\
\hline montevid & $-0.627^{* * *}(0.171)$ & $-0.240(0.063)$ \\
\hline siemprel & $0.537^{* * *}(0.171)$ & $0.211(0.066)$ \\
\hline intpol & $0.109(0.169)$ & \\
\hline homogene & $-0.511^{* * *}(0.191)$ & $.0 .191(0.067)$ \\
\hline activismo & $-0.325(0.178)$ & \\
\hline interven & $-0.387 * *(0.162)$ & $-0.151(0.063)$ \\
\hline autoidiz & $-0.977 * * *(0.267)$ & $-0.337(0.073)$ \\
\hline autoidde & $1.366^{* * *}(0.187)$ & $0.503(0.057)$ \\
\hline gestiong & $\cdot 1.245^{* * *}(0.175)$ & $.0 .464(0.059)$ \\
\hline econpais & $.0 .355(0.186)$ & \\
\hline econpers & $-0.494 * *(0.201)$ & $-0.184(0.070)$ \\
\hline impinf & $0.102(0.197)$ & \\
\hline infpub & $0.178(0.257)$ & \\
\hline
\end{tabular}

Al igual que para el modelo de voto a la familia de izquierda, el voto a la familia tradicional está fuertemente asociado a las dimensiones de valores políticos y satisfacción de intereses. La probabilidad predicha por el modelo de votar a la familia tradicional aumenta 50 puntos porcentuales entre quienes se ubican en la escala de autoidentificación ideológica en el espacio de derecha. Por su parte, el voto a los partidos tradicionales está vinculado negativamente con la autoidentificación de izquierda (la probabilidad estimada disminuye 34 puntos porcentuales).

En cuanto al efecto de la evaluación de la gestión de gobierno, la probabilidad de votar a la familia tradicional disminuye 46 puntos porcentuales si el encuestado evalúa positivamente la gestión del gobierno. Por su parte, la creencia de que el Estado debe aumentar el nivel de intervención en la economía, disminuye 15 puntos porcentuales la probabilidad estimada de votar a la familia tradicional, mientras que disminuye 18 puntos porcentuales cuando el encuestado evalúa positivamente su situación económica personal actual y futura.

\footnotetext{
${ }^{11}$ La probabilidad predicha por el modelo de que los encuestados respondan que votaron a la familia tradicional ( $f$ liatrad=1) es de 0,418 . Al tratarse de una variable dependiente binaria, esta probabilidad significa que en promedio un $41,8 \%$ de los entrevistados respondería que votó al Partido Nacional o al Partido Colorado. Mientras tanto, el porcentaje efectivo de personas que en la muestra manifestaron haber votado a la familia tradicional es de $46,1 \%$, por lo que la diferencia entre el valor predicho y el efectivo no es muy elevada (5,7\%). Por tanto, dicha medida de bondad de ajuste del modelo es satisfactoria aunque es una medida parcial.
} 
OPINIÃO PÚBLICA, Campinas, vol. 18, no 1, Junho, 2012, p. $197-215$

Pero entre votantes de la familia tradicional las características sociodemográficas tienen mayor peso y diversidad que en el voto al Frente Amplio. Según el modelo, el voto a la familia tradicional se vincula con la edad, la religión y el lugar de residencia. La probabilidad predicha por el modelo disminuye en 24 puntos porcentuales si el encuestado tiene entre 30 y 64 años y en 32 puntos porcentuales si tiene 65 o más años, lo que confirma la disminución del efecto demográfico en el voto por bloques (DE ARMAS, 2009; FLORES y SELIOS, 2011). Actualmente los jóvenes dividen más sus preferencias que en elecciones anteriores (MIERES, 2010). Quienes no creen en Dios tienen una probabilidad de 19 puntos porcentuales menos de votar al bloque tradicional en relación a quienes se declaran como creyentes y quienes no responden. Por su parte, el hecho de vivir en Montevideo, disminuye 24 puntos porcentuales la probabilidad predicha de votar a la familia tradicional, con respecto a quienes residen en el interior.

Otras variables que se asocian con el voto a la familia tradicional son: el haber votado al mismo partido en todas las elecciones en las que participó (asociación positiva), la homogeneidad en el voto personal y de familiares y amigos (asociación negativa). El hecho de haber votado al mismo partido en todas las elecciones aumenta 21 puntos porcentuales la probabilidad de votar a la familia tradicional. Por su parte, la probabilidad de votar a los partidos tradicionales disminuye 19 puntos porcentuales si el encuestado manifiesta homogeneidad entre su voto y el de su familia y amigos.

\section{Resumen de hallazgos de los modelos}

En resumen, según el primer modelo estimado, el voto al Frente Amplio en la elección de 2009 está fuertemente asociado a la percepción positiva de los individuos sobre la gestión del gobierno de Vázquez. La asociación con la evaluación de gestión de gobierno nos hace pensar que gran parte de la decisión del individuo que votó al Frente Amplio se basó en una evaluación de la performance del partido en el gobierno, de satisfacción de intereses, que se ve reflejada también en la asociación positiva con las variables de evaluación de la situación económica personal y del país.

Pero al mismo tiempo, la decisión electoral está asociada a valores políticos, especialmente un voto vinculado a lo ideológico en el sentido de que el voto al Frente Amplio se asocia positivamente con la autoidentificación de izquierda y negativamente con la autoidentificación de derecha. Al mismo tiempo, el voto al Frente Amplio se asocia positivamente con un valor asociado tradicionalmente a la autoidentificación de izquierda: el deseo de una mayor intervención del Estado en la economía.

Por otra parte, el modelo también muestra que el voto se vincula con variables de tipo comportamental. Así, el voto al Frente Amplio se asocia positivamente con el activismo político. Como ha sido señalado en la literatura, los votantes de este partido se caracterizan por participar en actos, caravanas y reuniones políticas en mayor medida que los votantes de otros partidos y quienes votan en blanco o anulado.

Algunos trabajos, (MOREIRA, 2000; CANZANI, 2000; MIERES, 1994) destacan que los frentistas estarían más informados que el promedio, algo más interesados en política y pertenecerían a clases de 
SELIOS, L.; VAIRO, D. Elecciones 2009 en Uruguay: permanencia de lealtades...

ingresos medios y altos con educación media y alta. Sin embargo, y más allá de que esas características hayan estado presentes en el pasado, la evidencia manejada en este trabajo muestra que no se manifestaron en 2009; ni el nivel educativo ni el interés en política se asocia al voto a ninguno de los dos bloques en Uruguay.

El voto al Frente Amplio en 2009 no se vincula con variables sociodemográficas, con la excepción de la asociación positiva con vivir en Montevideo, cuestión que ya ha sido ampliamente demostrada en otros trabajos y que resulta evidente al observar la información electoral.

Tampoco se vincula con haber votado siempre al mismo partido, lo que demuestra que el Frente Amplio durante varios años ha venido captando votantes de otros partidos, además de haber recibido por mucho tiempo nuevos votantes (BUQUET \& DE ARMAS, 2004).

En cuanto al voto a la familia tradicional, sí se vincula con características sociodemográficas de los encuestados. Se asocia en forma negativa con tener 30 años o más de edad. El hecho de que se asocie con esa franja etaria indica que el ser joven no es predictor de voto ni para el Frente Amplio ni para los partidos tradicionales, como ya veníamos señalando anteriormente. El voto a este bloque también se vincula negativamente con no creer en Dios y con residir en Montevideo. Tradicionalmente ambas variables solían distinguir los votantes nacionalistas de los colorados (MIERES, 1989).

Con respecto al resto de las variables, el voto a los partidos tradicionales se asocia significativamente a las mismas características y percepciones que las de los votantes del Frente Amplio, pero obviamente, con el signo contrario. La probabilidad de votar a los partidos tradicionales disminuye si la persona presenta homogeneidad en el voto de familia y amigos, si considera que debe intervenir más el Estado, si se autoidentifica como de izquierda y si evalúa positivamente su situación económica personal y la gestión del gobierno. En cambio, la probabilidad de votar al Partido Colorado o al Partido Nacional aumenta si el encuestado siempre votó al mismo partido y si se autoidentifica ideológicamente como de derecha.

En general, podemos concluir que el voto a las dos familias políticas principales en Uruguay en 2009 depende más de variables políticas como la ideología, la preferencia o no por un Estado más interventor y por variables de satisfacción de intereses (evaluación gestión de gobierno y de la situación económica), que variables de efecto de la campaña y la publicidad. Tampoco parece haber un efecto importante de la politización ni del nivel educativo entre los votantes de una y otra familia.

\section{¿Qué ha cambiado? Un análisis integrado interpretando el cambio electoral}

El análisis anterior nos informa de las determinantes del voto de los uruguayos en lo se ha definido aquí como el primer escalón de decisión, esto es, entre los bloques o familias políticas. Se ha visto que los hallazgos marcan continuidades y quiebres con lo que sabíamos sobre el voto en el país.

Dado que la familia de izquierda ha ensanchado su base electoral, las determinantes del voto han variado, así el voto al Frente Amplio ya no sólo se explica por la incorporación de nuevas generaciones, ni por el nivel educativo o el interés por la política, pero sí por aspectos ideológicos y de 
OPINIÃO PÚBLICA, Campinas, vol. 18, n 1, Junho, 2012, p. $197-215$

satisfacción de intereses, esto es evaluación de gestión y percepción económica del país ${ }^{12}$. En este trabajo, también aparece la percepción económica individual asociada al voto de la izquierda. Mientras que de las dimensiones "extra-políticas", el voto al FA mantiene una sola característica: el lugar de residencia.

Por su parte, en el voto a la familia tradicional también intervienen las variables que componen las dimensiones más "políticas", pero en la intención de voto a blancos y colorados aparecen varias características sociodemográficas y estructurales como son la religión, algunas categorías de edad y lealtad o pertenencia partidaria.

Si se analizan ambos modelos según el marco propuesto por (ROSE \& Mc ALLISTER, 1990), a fin de determinar la estabilidad/inestabilidad de los electores, se puede afirmar que el voto al Frente Amplio se ha tornado en este sentido más inestable, esto es, más sujeto a evaluaciones políticas que el voto a la familia tradicional, que aún presenta rasgos de factores estructurales. Esto indica que entre los votantes frenteamplistas se presentan componentes de inestabilidad: aparecen más sujetos a sus evaluaciones sobre gestión y economía que en el pasado. Posiblemente, éste componente se presente en los nuevos votantes frentistas, o sea, aquellos que pasaron de la familia tradicional a la de izquierda y no para los votantes leales que siguen existiendo.

Por el lado de la familia tradicional aún permanecen elementos de tipo inestables combinados con estables. Dicho más claramente, en el voto a la familia tradicional se combinan determinantes "extra políticos" con "políticos" casi por mitades. Así la evaluación de gestión, ideología y marcha de la economía son explicativas del voto junto con variables como el lugar de residencia, la creencia en dios y la fidelidad partidaria, aunque el poder explicativo de las últimas (medido a través de los efectos marginales) es menor. Esto puede explicar por qué dentro del bloque tradicional desde 1999 existe un traspaso de votos entre el Partido Colorado y el Partido Nacional elección tras elección. Por otro lado, los componentes de estabilidad nos permiten interpretar que son votantes leales a su bloque político, por lo que difícilmente serían votantes del Frente Amplio.

En resumen, en 2009 se observan electores leales a las familias ideológicas pero también, otras actitudes "políticas" - como evaluación de la gestión del gobierno, la percepción de la economía y los aspectos ideológicos- aparecen jugando fuertemente en esta elección caracterizada por la baja volatilidad. A su vez, ese nivel de volatilidad se puede entender por una posición bastante estable de los partidos y un segmento de electores inestables que moldean sus preferencias según aspectos "políticos"13.

En síntesis, esto muestra que por un lado las lealtades partidarias - basadas en elementos "extra políticos" consolidadas en las etapas de socialización primaria- son relevantes en las preferencias electorales de los uruguayos, pero también en esta elección, las actitudes "políticas". evaluación de la economía, y del gobierno. son relevantes, incorporándose así mecanismos de accountability electoral.

\footnotetext{
12 Resultados similares encuentra Canzani para la elección de 2004 (CANZANI, 2005).

${ }^{13}$ Consideramos que se consolida un fenómeno que comenzó en la elección de 2004: la evaluación sobre la economía, los resultados de políticas y las gestiones como predictores del voto a las familias políticas en el país.
} 


\section{Referencias Bibliográficas}

ADAMS, J.; MERRILL III, S. "Why Small, Centrist Third Parties Motivate Policy Divergence by Major Parties". American Political Science Review, v. 100, n. 3, p. 403.417, 2006.

AGUIAR, C. “La Historia y la historia: Opinión Pública y opinión pública en el Uruguay”. Prisma, v. 15, p. 7.45 , 2000.

ALTMAN, D. "Percepción ideológica de los partidos". Cuadernos del Claeh n. 85, p. 89-110, 2002.

ARMELLINI, M. Algunas notas sobre la evolución de las decisiones electorales en Uruguay. En: BUQUET, D. (coord.) Las claves del cambio. Ciclo electoral y nuevo gobierno 2004/2005. Montevideo: Ediciones de la Banda Oriental, 2005.

BUQUET, D.; DE ARMAS, G. La evolución electoral de la izquierda: crecimiento demográfico y moderación ideológica. En: LANZARO, J. (coord). La izquierda uruguaya entre la oposición y el gobierno. Montevideo: Editorial Fin de Siglo, 2004.

BUQUET, D.; JOHNSON, N. (eds.). Del cambio a la continuidad: Ciclo electoral 2009-2010 en Uruguay. Montevideo: Ed. Banda Oriental, 2010.

BUQUET, D.; SELIOS, L. "El escenario preelectoral y la Opinión Pública", Informe de coyuntura, n. 5, Instituto de Ciencia Política. Montevideo: Ediciones de la Banda Oriental, 2004.

BUQUET, D. Elecciones uruguayas 2004-2005: De la vieja oposición a la nueva mayoría. En: BUQUET, D. (coord.): Las claves del cambio. Ciclo electoral y nuevo gobierno 2004/2005. Montevideo: Ediciones de la Banda Oriental, 2005.

CANZANI, A. Mensaje en una botella: analizando las elecciones de 1999-2000. Elecciones 1999-2000. Montevideo. Colección política Viva, Instituto de Ciencia Política, Universidad de la república. Ediciones de la Banda Oriental, 2000.

Cómo llegar a buen puerto: un análisis desde la opinión pública de la trayectoria electoral del EPFA. En: BUQUET, D. (coord.). Las claves del cambio. Ciclo electoral y nuevo gobierno 2004/2005. Montevideo, Ediciones de la Banda Oriental, 2005.

CHASQUETTI, D. "Compartiendo el gobierno multipartidismo y coaliciones en el Uruguay (1971-1997)". Revista Uruguaya de Ciencia Política, n.10, 1998.

CHASQUETTI, D; GARCÉ, A. "Unidos por la historia: Desempeño electoral y perspectivas de colorados y blancos como bloque político". En: BUQUET, D. (coord.). Las claves del cambio. Ciclo electoral y nuevo gobierno 2004/2005. Montevideo: Ediciones de la Banda Oriental, 2005

COLOMER, J; ESCATEL, L. "La dimensión izquierda y derecha en América Latina". Desarrollo Económico v. 45, n. 177, p. $123-136,2005$.

DE ARMAS, G. Autopsia de los votantes: los efectos de la campaña en la decisión electoral". En: BUQUET, D. (coord.). Las claves del cambio. Ciclo electoral y nuevo gobierno 2004/2005. Montevideo: Ediciones de la Banda Oriental, 2005.

"Debilitamiento del efecto demográfico y consolidación de un nuevo sistema de partidos: evidencia de las elecciones 2009 en Uruguay". Revista Uruguaya de Ciencia Política, v. 18, n.1, 2009.

DOWNS, A. An Economic Theory of Democracy. Nueva Cork: Harper and Row, 1957.

FIORINA, M. Retrospective voting in American Presidential election. Yale: University Press, 1981. 1997.

Voting Behavior. In: MUELLER, D. (ed). Perspectives on Public Choice. Cambridge: Cambridge University Press,

FLORES, M.; SELIOS, L. "Perfiles generacionales en las preferencias políticas de los uruguayos". Revista Uruguaya de Ciencia Política, v. 20, n. 1, 2011

GARCÉ, A.; YAFFÉ, J. La era progresista. Montevideo: Editorial Fin de Siglo, 2004.

GONZÁLEZ, L.E. Estructuras políticas y democracia en Uruguay. Montevideo: Fundación de Cultura Universitaria, 1993.

. Los partidos establecidos y sus desafiantes. En: GONZÁLEZ, L.E. Los partidos políticos uruguayos en tiempos de cambio. Montevideo: Fundación de Cultura Universitaria, 1999. 
OPINIÃO PÚBLICA, Campinas, vol. 18, no 1, Junho, 2012, p. $197-215$

“¿Los uruguayos se corren a la izquierda?”, Semanario Búsqueda, p. 19, 30 set.2004.

GONZÁLEZ, L.E.; QUEIROLO, R. Las Elecciones Nacionales del 2004: posibles escenarios, en Instituto de Ciencia Política: Elecciones 1999-2000. Colección política Viva, Instituto de Ciencia Política, Universidad de la República. Montevideo: Ediciones de la Banda Oriental, 2000.

HARETCHE, C. Cultura política y democracia en el Uruguay. Documento de Trabajo ${ }^{\circ} 44$. Montevideo: Instituto de Ciencia Política, 2004.

INGLEHART, R. From class-based to value-based politics. En: MAIR, P. (ed.). The west european party system. Oxford: Oxford University Press, 1990.

KRIESI, H. "The transformation of cleavage politics. The 1997 Stein Rokkan lecture". European Journal of Political Research, n. 33, 1998.

LAZARSFELD, P.; BERELSON, B.; GAUDET, H. The people's choice. New York: Columbia University Press, 1948

LANZARO, J. “EI Presidencialismo pluralista en la Segunda Transición”, En: LANZARO, J. (Coord.). La segunda Transición en Uruguay. Montevideo: Fundación de Cultura Universitaria, 2000.

LIPSET, S. M. El hombre político. Buenos Aires: Editorial Tecnos·Rei, 1988.

LUNA, J.P. “¿Pesimismo estructural o voto económico?” Macropolitics in: Uruguay. Revista Uruguaya de Ciencia Política, n.13, 2002.

LUNA, J. P. "De familias y parentescos políticos: ideología y competencia electoral en el Uruguay contemporáneo". En: LANZARO, J. (coord) : La izquierda uruguaya entre la oposición y el gobierno. Montevideo: Editorial Fin de Siglo, 2004.

MAGRI, A. "A la mesa, Canelones está servido". Instituto de Ciencia Política: Informe de coyuntura, Montevideo: Ediciones de la Banda Oriental, n.5, 2004.

MARAVALL, J. M. y SAMCJEZ-CUENCA, I. (eds.). Controlling Governments Voters, Institutions, and Accontability. Cambridge: Cambridge University Press, 2008.

MIERES, P. ¿Cómo votan los uruguayos? Las elecciones de 1984. Montevideo: Ediciones De la Banda Oriental, 1988.

"Elecciones de 1989: el cambio del sistema de partidos y las adhesiones politicas de los uruguayos". En: Partidos y electores. Centralidad y cambio. Montevideo: Colección Argumentos, Claeh, n. 17, 1990.

El voto en el Uruguay de fin de sig/o. Montevideo: Editorial Fin de Siglo, 1994. 1997.

Intermediación política y cambio electoral: algunas líneas de interpretación. Cuadernos de CLAEH, n.78-79,

La edad y el cambio electoral en Uruguay, 2010. En: ZUASNABAR, I.; GONZALEZ, L. E.; MIERES, P.; IRAZABAL, F. (eds.). El voto en el Uruguay 2009-2010. Montevideo: Universidad Católica del Uruguay y Fundación Konrad Adenauer, 2010.

MONESTIER, F. Familia e identidad partidaria. Razones para el éxito de una nueva transformación en Uruguay. En: I SEMINARIO REGIONAL DE WAPOR, Punta del Este, 1999.

MOREIRA, C. Comportamiento electoral y cultura política. Colección política Viva, Instituto de Ciencia Política, Universidad de la república. Elecciones 1999/2000. Montevideo: Ediciones de la Banda Oriental, 2000. 2004.

Final de juego. Del bipartidismo tradicional al triunfo de la izquierda en Uruguay. Montevideo: Ediciones Trilce,

El voto moderno y el voto clasista revisado; explicando el desempeño electoral de la izquierda en las elecciones de 2004 en Uruguay. En: BUQUET, D. (coord.). Las claves del cambio. Ciclo electoral y nuevo gobierno 2004/2005. Montevideo: Ediciones de la Banda Oriental, 2005.

OSKAMP, S. Attitudes and opinions. New Jersey: Prentice Hall, 1991. 
SELIOS, L.; VAIRO, D. Elecciones 2009 en Uruguay: permanencia de lealtades...

PARAMIO, L. "Clase y voto: intereses, identidades y preferencias". Revista Española de investigaciones Sociológicas, n. 90, Centro de Investigaciones Sociológicas, Madre, 2000.

PIZZORNO, A. Sobre la racionalidad de la opción democrática. En: PIZZORNO, A. et al (orgs.). Los límites de la democracia. v. 2. Buenos Aires: CLACSO, 1985.

POPKIN, S. The reasoning voter. Chicago: The University of Chicago Press, 1994.

POWELL, B. JR. Elections as Instruments of democracy: Majoritarian and proportional visions. Yale: Yale University Press, 2000.

QUEIROLO, R. La "tradicionalización" del Frente Amplio: La conflictividad del proceso de cambio. En: GONZÁLES, F.;

MONESTIER. F.; QUEIROLO, R.; RICO, M. S. (orgs.). Los partidos políticos uruguayos en tiempos de cambio. Montevideo: Fundación de Cultura Universitaria, 1999.

"Las elecciones uruguayas de 2004: la izquierda como la única oposición creíble". Revista Colombia Internacional. ก. 64,2006

ROSE, R.; Mc ALLISTER, I. The loyalties of voters. SAGE Publications, 1990.

ROSELL, C. “Tipos democráticos y opinión pública en el Uruguay”. Revista Uruguaya de Ciencia Política, n. 13, 2002.

SELIOS, L. Opinión Pública, 2009. Encrucijada 2010. La política uruguaya a prueba, Informe de Coyuntura. N. 9, Instituto de Ciencia Política, Classo Colecciones. Montevideo: Editorial Fin de Siglo, 2009.

SOTELO, M. "La longevidad de los partidos tradicionales uruguayos desde una perspectiva comparada", En: GONZÁLES, F.; MONESTIER. F.; QUEIROLO, R.; RICO, M. S. (orgs.). Los partidos políticos uruguayos en tiempos de cambio. Montevideo: Fundación de Cultura Universitaria, 1999.

YAFFÉ, J. Réquiem para el Réquiem para la izquierda. El triunfo del FA: de la competencia intrapartidaria al desempeño electoral, En: BUQUET, D. (coord.). Las claves del cambio. Ciclo electoral y nuevo gobierno 2004/2005. Montevideo: Ediciones de la Banda Oriental, 2005.

Lucía Selios·Iselios@gmail.com

Daniela Vairo·danielavairo@gmail.com

Recebido para publicação em abril de 2011. Aprovado para publicação em dezembro de 2011. 